\title{
Throughput Maximization for Buffer-Aided Hybrid Half-/Full-Duplex Relaying with Self-Interference
}

\author{
Mohammad Galal Khafagy ${ }^{\S}$, Ahmed El Shafie*, Ahmed Sultan ${ }^{\S}$, and Mohamed-Slim Alouini ${ }^{\S}$ \\ $\S$ King Abdullah University of Science and Technology (KAUST), Thuwal, Makkah Province, Saudi Arabia. \\ * Electrical Engineering Department, University of Texas at Dallas, USA. \\ Email: mohammad.khafagy@kaust.edu.sa, ahmed.elshafie@utdallas.edu, \{ahmed.salem, slim.alouini\}@kaust.edu.sa
}

\begin{abstract}
In this work, we consider a two-hop cooperative setting where a source communicates with a destination through an intermediate relay node with a buffer. Unlike the existing body of work on buffer-aided half-duplex relaying, we consider a hybrid half-/full-duplex relaying scenario with loopback interference in the full-duplex mode. Depending on the channel outage and buffer states that are assumed available at the transmitters, the source and relay may either transmit simultaneously or revert to orthogonal transmission. Specifically, a joint source/relay scheduling and relaying mode selection mechanism is proposed to maximize the end-to-end throughput. The throughput maximization problem is converted to a linear program where the exact global optimal solution is efficiently obtained via standard convex/linear numerical optimization tools. Finally, the theoretical findings are corroborated with event-based simulations to provide the necessary performance validation.
\end{abstract}

Keywords-buffer-aided relay, full-duplex, linear-fractional programming, outage, queuing theory, self-interference, throughput.

\section{INTRODUCTION}

Motivated by the current surge in mobile internet, the International Telecommunication Union (ITU) has recently begun to set the stage for fifth generation (5G) mobile network specifications. Despite being still underway, communications experts from both industry and academia have already started to discuss the set of challenges 5G networks are envisioned to tackle [1], [2]. This includes the support of different traffic types with diverse demands, better sustainability via reduced energy consumption, along with higher quality of user experience. To meet such challenges, next generation networks are anticipated to adopt an extensive reshaping of its infrastructure along with employing novel radio technologies that offer high efficiency from both spectral and energy perspectives. For instance, an infrastructure densification is expected in which heterogeneous deployments of macrocells, picocells, and relays are jointly utilized to enhance the end-user experience [1]. Moreover, beyond the currently employed conventional half-duplex radio, supporting simultaneous transmission and reception over the same channel via in-band full-duplex transceivers represents a strong radio technology candidate [2]. In this work, our attention is focused on leveraging full-duplex operation for end-to-end throughput maximization.

Buffer-aided relaying was proposed in [3] in order to boost the capacity of half-duplex decode-and-forward (DF) relay channels, which is known to be limited by the minimum capacity of the first and second hops. The impact of packet loss at the relay due to a severely attenuated relay-destination link is mitigated by the ability to queue data until the link becomes reliable. Further capacity gains were demonstrated by Zlatanov et al. in [4] via the relaxation of the round-robin listening/forwarding phase allocation and adopting instead an adaptive link selection approach based on the instantaneous quality of the two hops. Despite these recent efforts, all the existing work has only addressed buffer-aided half-duplex relaying $(H D R)$.

After the recent developments and first implementations of full-duplex nodes [5], [6], full-duplex relaying (FDR) received considerable attention from the community. Specifically, efforts were directed to the performance analysis and optimization of FDR channels, for both cooperative scenarios targeting coverage extension and/or throughput enhancement such as in [7], [8] and the references therein. This was motivated by the allowed simultaneous source/relay transmission in FDR, which avoids the rate penalty experienced by HDR due to resource-orthogonal listening/forwarding. However, even after the employment of sophisticated isolation and cancellation techniques [5], [6], FDR suffers from a residual level of selfinterference due to the simultaneous transmission/reception at the relay. In [7], the capacities of HDR and FDR channels were compared, and it was shown that they exchange performance superiority over different channel configurations. Accordingly, a hybrid bufferless HDR/FDR protocol was proposed in [7] to switch between the two relaying modes and attain a higher performance than each individual protocol.

In this work, buffer-aided FDR with self-interference is studied for the first time to the best of the authors' knowledge. We focus on coverage extension scenarios where the direct source-destination link is assumed unavailable due to long distance and severe shadowing effects. The source adopts a fixed rate transmission which renders each fading link in one of only two possible states; either in outage or not. A queuing theoretic approach is adopted in order to investigate the possible end-toend throughput gains. First, assuming both buffer and outage state information are available at the transmitters, we propose a hybrid HDR/FDR protocol that jointly schedules source/relay transmissions and controls relaying mode switching. Based on the link outage state, the protocol selects to either adopt FDR or revert to HDR due to the adverse effect of selfinterference. Further, when the HDR mode is selected, the protocol selects whether the source or the relay accesses the channel based on the buffer state. A throughput maximization problem is formulated and converted to an efficiently solvable linear program using standard numerical optimization tools. Second, we investigate the throughput when the buffer state information (BSI) is not provided. Finally, we compare the theoretical results of the proposed scheme to conventional bufferless HDR and FDR, and validate them with results 


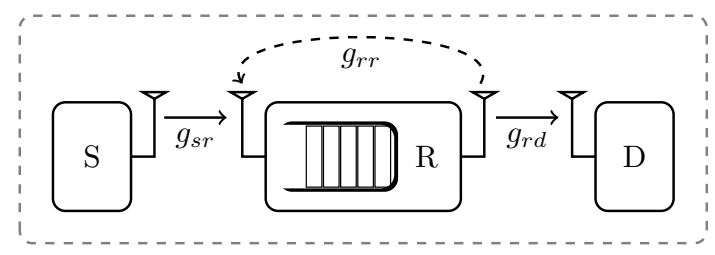

Fig. 1. A buffer-aided full-duplex relay channel with self-interference.

obtained via event-based simulations.

The rest of the paper is organized as follows. We explain the studied system model in section II, while the scheduling protocol is proposed in section III. The throughput maximization problem is formulated and solved in section IV. The theoretical findings of this work is validated in section $\mathrm{V}$ by comparison with the results obtained via numerical simulations. Finally, conclusions are drawn in section VI.

\section{SYSTEM MODEL}

\section{A. Channel Model}

We study the two-hop cooperative setting depicted in Fig. 1 , where a source $S$ communicates with a destination $D$ via a $\mathrm{DF}$ relay $\mathrm{R}$. The relay $\mathrm{R}$ is equipped with a buffer of size $N$ data packets, and suffers a residual level of self-interference while operating in a full-duplex mode. As shown, $g_{s r}, g_{r d}$ and $g_{r r}$ denote the fading gains, i.e., the squared magnitude of the complex fading coefficients, for the $S-R, R-D$ and $R-R$ links, respectively. The channels are assumed to experience block fading, where the channel gains remain constant over one block, and vary independently from one block to another following a general probability distribution, i.e., the analysis to follow is not limited to a specific fading model. Moreover, we assume that the direct source-destination link is unavailable due to large-distance and shadowing effects. The received signals at the relay and the destination are perturbed by additive white Gaussian noise with zero mean and variance $\sigma_{r}^{2}$ and $\sigma_{d}^{2}$, respectively.

\section{B. Signal Model}

We assume that the source adopts a fixed rate transmission with a source rate of $\mathcal{R}$ bits/sec/Hz, and that the transmission power is fixed for the source and the relay to $P_{s}$ and $P_{r}$, respectively. The source seeks to adopt the best access scheme which can maximize the end-to-end throughput. It is assumed that the BSI is known at the source via acknowledgement/negative acknowledgement (ACK/NACK) messages from the relay, like for instance in [9]. Also, channel state information (CSI) is assumed available at the transmitters, not necessarily of the exact channel state, but only of its outage state. This can be provided, for instance, via one-bit feedback messages. Finally, the link outage probabilities are calculated at the transmitters with their knowledge of the transmission rate and the channel statistics.

The relay is assumed to adopt a hybrid HDR/FDR scheme according to the link outage states. For instance, when the $\mathrm{S}-\mathrm{R}$ link undergoes an outage while the relay is active due to loopback interference, time-orthogonal transmission, i.e., HDR, may still take place. In this channel state, the

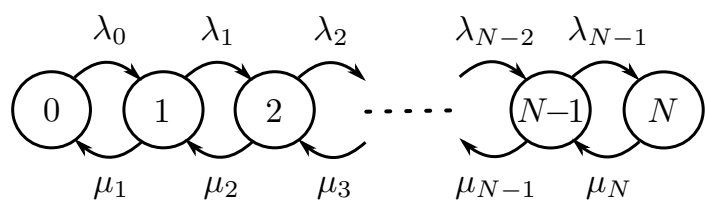

Fig. 2. Birth-death Markov process. Transitions from a state to itself are omitted for visual clarity.

relay may either individually access the channel to forward the accumulated packets in its queue, or go into a listenonly mode while the source transmits. The latter case gives a higher chance for successful packet delivery from the source to the relay by eliminating the loopback interference effect. As commonly assumed in the literature, an RF-chain conserved scenario [10] is assumed when switching between HDR and FDR, i.e., the relay is equipped with exactly one transmit and one receive RF-chains. Therefore, single-antenna HDR is only possible when reverting to orthogonal transmission.

The received signal-to-interference-plus-noise ratio (SINR) at the relay when it is silent or active is given respectively by

$$
\gamma_{1}=\frac{P_{s} g_{s r}}{\sigma_{r}^{2}}, \quad \text { and } \quad \gamma_{2}=\frac{P_{s} g_{s r}}{P_{r} g_{r r}+\sigma_{r}^{2}} .
$$

Also, the received SINR at the destination is given by

$$
\gamma_{3}=\frac{P_{r} g_{r d}}{\sigma_{d}^{2}} .
$$

We use $\mathcal{O}_{i}$ for $i \in\{1,2,3\}$ to denote a link outage event where the link capacity $\log _{2}\left(1+\gamma_{i}\right)$ falls below the source rate $\mathcal{R}$, while $\mathcal{P}_{i}$ denotes the probability of occurrence of $\mathcal{O}_{i}$. Throughout the manuscript, $\overline{\mathcal{O}_{i}}$ will be used to denote the complementary event of $\mathcal{O}_{i}$. Also, $\overline{\mathcal{P}_{i}}=1-\mathcal{P}_{i}$. The different link outage events are summarized in Table I, with $\mathbb{P}\{A\}$ denoting the probability of occurrence of the event $A$.

\section{TRANSMission SCHEdUling PROTOCOL}

The source node is assumed to adopt a scheduling scheme where its access to the channel depends on the available BSI and CSI. A handshaking mechanism is assumed available between the source and the relay, which can take place, for instance, via a dedicated low-rate control channel. The relay node is equipped with a buffer that can store up to $N$ packets. The queuing system under investigation can be modeled as a birth-death Markov chain as shown in Fig. 2, where the different states represent the number of packets in the relay's queue. Let $\epsilon_{n}$ denote the steady-state probability of the queue being in state $n$, while $\lambda_{n}$ and $\mu_{n}$ respectively denote the state increment and decrement probability when the queue is at state $n$. In what follows, we carefully explain the proposed scheduling scheme.

The probability space is partitioned into three mutually exclusive events, namely, (i) $\mathcal{O}_{1}$, (ii) $\mathcal{O}_{2} \cap \overline{\mathcal{O}_{1}}$, and (iii) $\overline{\mathcal{O}_{2}}$. Note that $\mathcal{O}_{1} \subset \mathcal{O}_{2}$, and hence, the probability of $\mathcal{O}_{2} \cap \overline{\mathcal{O}_{1}}$ is $\mathcal{P}_{2}-\mathcal{P}_{1}=\overline{\mathcal{P}_{1}}-\overline{\mathcal{P}_{2}}$. It can be easily verified that the probabilities of these three events sum up to 1 . The proposed scheduling protocol is defined over these events as follows.

(i) When the $\mathrm{S}-\mathrm{R}$ link is in outage even with a silent relay, i.e., in the $\mathcal{O}_{1}$ event, the source remains silent while the 
TABLE I. DEFINITION OF OUTAGE EVENTS

\begin{tabular}{|c|c|c|}
\hline Event & Probability & Definition \\
\hline $\mathcal{O}_{1}$ & $\mathcal{P}_{1}=\mathbb{P}\left\{\log _{2}\left(1+\frac{P_{s} g_{s r}}{\sigma_{r}^{2}}\right)<\mathcal{R}\right\}$ & Probability of $\mathrm{S}-\mathrm{R}$ link outage with a silent relay \\
\hline $\mathcal{O}_{2}$ & $\mathcal{P}_{2}=\mathbb{P}\left\{\log _{2}\left(1+\frac{P_{s} g_{s r}}{P_{r} g_{r r}+\sigma_{r}^{2}}\right)<\mathcal{R}\right\}$ & Probability of S $-\mathrm{R}$ link outage with an active relay \\
\hline $\mathcal{O}_{3}$ & $\mathcal{P}_{3}=\mathbb{P}\left\{\log _{2}\left(1+\frac{P_{r} g_{r d}}{\sigma_{d}^{2}}\right)<\mathcal{R}\right\}$ & Probability of R - D link outage \\
\hline
\end{tabular}

TABLE II. Proposed Transmission Scheduling Protocol

\begin{tabular}{|c|c|c|c|c|c|}
\hline \multirow{2}{*}{ (i) $\mathcal{O}_{1}$} & \multicolumn{2}{|c|}{ (ii) $\mathcal{O}_{2} \cap \overline{\mathcal{O}_{1}}$} & \multicolumn{2}{|c|}{ (iii) $\overline{\mathcal{O}_{2}}$} \\
\cline { 3 - 6 } & always silent & (a) $\mathcal{O}_{3}$ & (b) $\overline{\mathcal{O}_{3}}$ & (a) $\mathcal{O}_{3}$ & (b) $\overline{\mathcal{O}_{3}}$ \\
\hline Source & at non-full buffer & w.p. $\beta_{n}$ & at non-full buffer & $\begin{array}{c}\text { (I) at non-full buffer } \\
\text { (II) always active }\end{array}$ \\
\hline Relay & at $\overline{\mathcal{O}_{3}}$ with non-empty buffer & always silent & w.p. $\overline{\beta_{n}}$ & always silent & at non-empty buffer \\
\hline
\end{tabular}

relay accesses the channel when the $\mathrm{R}-\mathrm{D}$ link is not in outage and its buffer is non-empty.

(ii) When the $\mathrm{S}-\mathrm{R}$ link is in outage if the relay is active while it is not when the relay is silent, i.e., in $\mathcal{O}_{2} \cap \overline{\mathcal{O}_{1}}$, the scheme further depends on the $\mathrm{R}-\mathrm{D}$ link outage state.

(a) When the $\mathrm{R}-\mathrm{D}$ link is in outage, i.e., in $\mathcal{O}_{3}$, only the source can transmit at non-full relaying buffer.

(b) When the $\mathrm{R}-\mathrm{D}$ link is not in outage, i.e., in $\overline{\mathcal{O}_{3}}$, the source and relay transmit in orthogonal time slots due to the loopback interference adverse effect on the $\mathrm{S}-\mathrm{R}$ link. Moreover, the access probability will depend on the state of the relay. Let the probability that the source transmits be $\beta_{n}$ when the buffer has $n$ packets, while the relay transmits with probability $\overline{\beta_{n}}=1-\beta_{n}$. It is clear that $\beta_{0}=1$ and $\beta_{N}=0$ when BSI is available.

(iii) When the $\mathrm{S}-\mathrm{R}$ link is not in outage even with an active relay, i.e., in $\overline{\mathcal{O}_{2}}$, also the transmission scheme further depends on the $\mathrm{R}-\mathrm{D}$ link outage state.

(a) In $\mathcal{O}_{3}$, the relay remains silent, while the source transmits provided that the relay's buffer is not full.

(b) In $\overline{\mathcal{O}_{3}}$, the source and relay can simultaneously transmit, taking advantage of FDR capability. Of course, the relay transmits when its buffer is nonempty. There are two slightly different variants of the protocol which can be adopted by the source though. These two variants stem from whether or not an active full-duplex relay accepts a packet while its queue is full. Specifically, in variant $\mathrm{I}$, the relay can be assumed to reject any packets when its queue is in state $N$, which yields that the source transmits in $1-\epsilon_{N}$ of the time only (when the buffer is not full). On the other hand, due to FDR, it can be assumed in variant II that the relay has an additional onepacket transmission buffer. If it transmits, the relay will take out the packet at the head of its main queue at the beginning of the time slot and put it in the transmission buffer. The source can then transmit concurrently with the relay even at a full-buffer state, since there is always a room for an upcoming packet.

The above proposed transmission scheduling protocol is summarized in Table II.
Let $x(t)$ denote the queue state at time $t$. A state increment occurs due to one of the events (ii)-(a), (ii)-(b), (iii)-(a), and (iii)-(b) with probability

$$
\begin{aligned}
\lambda_{n}= & \mathbb{P}\{x(t)=n+1 \mid x(t-1)=n\} \\
= & \overline{\mathcal{P}_{1}} \mathcal{P}_{3}+\left(\overline{\mathcal{P}_{1}}-\overline{\mathcal{P}_{2}}\right) \overline{\mathcal{P}_{3}} \beta_{n}+\overline{\mathcal{P}_{2}} \overline{\mathcal{P}_{3}} \delta_{n, 0}, \\
& \delta_{n, j}= \begin{cases}1 & \text { if } n=j \\
0 & \text { otherwise }\end{cases}
\end{aligned}
$$

The last term in (3) comes from the fact that a state increment occurs in the event (iii)-(b) only when the relay does not have packets in its queue to forward. Otherwise, simultaneous arrival/departure occurs in the full-duplex mode which contributes to the system throughput, however it does not alter the state of the queue. Also, the first term in (3) comes from the sum of the probabilities in the events (ii)-(a) and (iii)-(a).

\section{A. Variant I}

A state decrement occurs due to the events (i), (ii)-(b), and (iii)-(b) with probability

$$
\begin{aligned}
\mu_{n} & =\mathbb{P}\{x(t)=n-1 \mid x(t-1)=n\} \\
& =\mathcal{P}_{1} \overline{\mathcal{P}_{3}}+\left(\overline{\mathcal{P}_{1}}-\overline{\mathcal{P}_{2}}\right) \overline{\mathcal{P}_{3}} \overline{\beta_{n}}+\overline{\mathcal{P}_{2}} \overline{\mathcal{P}_{3}} \delta_{n, N} \\
& =\mathcal{P}_{2} \overline{\mathcal{P}_{3}}-\left(\overline{\mathcal{P}_{1}}-\overline{\mathcal{P}_{2}}\right) \overline{\mathcal{P}_{3}} \beta_{n}+\overline{\mathcal{P}_{2}} \overline{\mathcal{P}_{3}} \delta_{n, N} .
\end{aligned}
$$

The source throughput is then given from the events (ii)-(a), (ii)-(b), (iii)-(a), and (iii)-(b) as

$$
\mathcal{T}_{\mathrm{I}}=\left(\overline{\mathcal{P}_{1}}-\overline{\mathcal{P}_{2}}\right) \overline{\mathcal{P}_{3}} \sum_{n=0}^{N-1} \epsilon_{n} \beta_{n}+\left(\overline{\mathcal{P}_{1}} \mathcal{P}_{3}+\overline{\mathcal{P}_{2}} \overline{\mathcal{P}_{3}}\right)\left(1-\epsilon_{N}\right),
$$

where the first term in (7) comes from the (ii)-(b) event over non-full queue states, while the second term comes from the sum of the throughput components in the rest of the events also at non-full queue states.

\section{B. Variant II}

If we assume that there is always a room for a packet when the relay is active regardless of the buffer state, a queue state can never be decremented in the event of simultaneous source/relay transmissions, i.e., in (iii)-(b). However, this event still contributes to the throughput of the system. Hence, in 
variant II, the state decrement probability and the throughput become

$$
\begin{aligned}
\mu_{n} & =\mathcal{P}_{1} \overline{\mathcal{P}_{3}}+\left(\overline{\mathcal{P}_{1}}-\overline{\mathcal{P}_{2}}\right) \overline{\mathcal{P}_{3}} \overline{\beta_{n}} \\
& =\mathcal{P}_{2} \overline{\mathcal{P}_{3}}-\left(\overline{\mathcal{P}_{1}}-\overline{\mathcal{P}_{2}}\right) \overline{\mathcal{P}_{3}} \beta_{n}
\end{aligned}
$$

and

$$
\mathcal{T}_{\text {II }}=\left(\overline{\mathcal{P}_{1}}-\overline{\mathcal{P}_{2}}\right) \overline{\mathcal{P}_{3}} \sum_{n=0}^{N-1} \epsilon_{n} \beta_{n}+\overline{\mathcal{P}_{1}} \mathcal{P}_{3}\left(1-\epsilon_{N}\right)+\overline{\mathcal{P}_{2}} \overline{\mathcal{P}_{3}}
$$

For both variants, the local balance equations of the birth-death Markov chain in Fig. 2 are given by

$$
\epsilon_{n} \lambda_{n}=\epsilon_{n+1} \mu_{n+1}, \text { for } 0 \leq n \leq N-1,
$$

or

$$
\epsilon_{n+1}=\frac{\lambda_{n}}{\mu_{n+1}} \epsilon_{n}=\prod_{k=0}^{n} \frac{\lambda_{k}}{\mu_{k+1}} \epsilon_{0}, \text { for } 0 \leq n \leq N-1 .
$$

Since $\sum_{n=0}^{N} \epsilon_{n}=1$, then we can obtain $\epsilon_{0}$ as

$$
\epsilon_{0}=\left(1+\sum_{n=1}^{N} \prod_{k=0}^{n-1} \frac{\lambda_{k}}{\mu_{k+1}}\right)^{-1} .
$$

It is worth noting that the end-to-end throughput can be equally calculated as either originating from the source, as given in (7) and (10), or arriving at the destination. This can be shown by summing both sides of the local balance equation in (11) from $n=0$ to $N-1$. Substituting back in (7) and (10) yields the alternative expressions derived from the destination's perspective in the events (i), (ii)-(b) and (iii)-(b). In the following section, we design the access probabilities, $\left\{\beta_{n}\right\}_{n=0}^{N}$, to maximize the end-to-end throughput.

\section{Throughrut MaXimization}

When both the BSI and CSI are available at the transmitters, the end-to-end throughput maximization problem can be formally written, for $i \in\{\mathrm{I}, \mathrm{II}\}$, as

$$
\begin{aligned}
\max _{\left\{\beta_{n}\right\}_{n=0}^{N}} & \mathcal{T}_{i} \\
\text { s.t. } & \beta_{0}=1, \quad \beta_{N}=0, \\
& 0 \leq \beta_{n} \leq 1, \quad \text { for } n=1,2, \ldots, N-1 .
\end{aligned}
$$

\section{A. Variant I}

We first define a new set of variables $\left\{\Psi_{n}\right\}_{n=1}^{N}$ as

$$
\Psi_{n}=\frac{\epsilon_{n}}{\epsilon_{0}}=\prod_{k=0}^{n-1} \frac{\lambda_{k}}{\mu_{k+1}} .
$$

It is clear that $\Psi_{0}=\frac{\epsilon_{0}}{\epsilon_{0}}=1$. We rewrite the throughput expression by substituting (12)-(15) into (7) as

$$
\mathcal{T}_{\mathrm{I}}=\left(\overline{\mathcal{P}_{1}} \mathcal{P}_{3}+\overline{\mathcal{P}_{2}} \overline{\mathcal{P}_{3}}\right)+\phi,
$$

where

$$
\phi=\frac{\left(\overline{\mathcal{P}_{1}}-\overline{\mathcal{P}_{2}}\right) \overline{\mathcal{P}_{3}} \sum_{n=0}^{N-1} \Psi_{n} \beta_{n}-\left(\overline{\mathcal{P}_{1}} \mathcal{P}_{3}+\overline{\mathcal{P}_{2}} \overline{\mathcal{P}_{3}}\right) \Psi_{N}}{\left(1+\sum_{n=1}^{N} \Psi_{n}\right)} .
$$

The new variables $\left\{\Psi_{n}\right\}_{n=1}^{N}$ can be written in terms of the old variables $\left\{\beta_{n}\right\}_{n=0}^{N}$ by substituting (3) and (6) into (15) as
$\Psi_{n}=\prod_{k=0}^{n-1} \frac{\overline{\mathcal{P}_{1}} \mathcal{P}_{3}+\left(\overline{\mathcal{P}_{1}}-\overline{\mathcal{P}_{2}}\right) \overline{\mathcal{P}_{3}}-\left(\overline{\mathcal{P}_{1}}-\overline{\mathcal{P}_{2}}\right) \overline{\mathcal{P}_{3}} \beta_{k+1}+\overline{\mathcal{P}_{2}} \overline{\mathcal{P}_{2}} \overline{\mathcal{P}}_{3} \delta_{k, 0}}{\overline{\mathcal{P}_{3}} \delta_{k+1, N}}$.

Maximizing $\mathcal{T}_{\mathrm{I}}$ is equivalent to maximizing $\phi$. Moreover, we can notice in (17) that $\phi$ can be put in the form of a linearfractional function in $\left\{\Psi_{n}\right\}_{n=1}^{N}$ if we manage to express $\left\{\Psi_{n} \beta_{n}\right\}_{n=0}^{N-1}$ in terms of $\left\{\Psi_{n}\right\}_{n=1}^{N}$ only. We have

$$
\frac{\Psi_{n}}{\Psi_{n-1}}=\frac{\overline{\mathcal{P}_{1}} \mathcal{P}_{3}+\left(\overline{\mathcal{P}_{1}}-\overline{\mathcal{P}_{2}}\right) \overline{\mathcal{P}_{3}} \beta_{n-1}+\overline{\mathcal{P}_{2}} \overline{\mathcal{P}_{3}} \delta_{n-1,0}}{\mathcal{P}_{2} \overline{\mathcal{P}_{3}}-\left(\overline{\mathcal{P}_{1}}-\overline{\mathcal{P}_{2}}\right) \overline{\mathcal{P}_{3}} \beta_{n}+\overline{\mathcal{P}_{2}} \overline{\mathcal{P}_{3}} \delta_{n, N}} .
$$

Hence, we get the recurrence relation

$$
\beta_{n} \Psi_{n}=a_{n} \Psi_{n}-b_{n-1} \Psi_{n-1}-\beta_{n-1} \Psi_{n-1},
$$

with its initial value given by

$$
\beta_{1} \Psi_{1}=a_{1} \Psi_{1}-b_{0}-\beta_{0},
$$

where

$$
a_{n}=\left\{\begin{array}{l}
\frac{1}{\left(\overline{\mathcal{P}_{1}}-\overline{\mathcal{P}_{2}}\right)}, n=N, \\
\frac{\mathcal{P}_{2}}{\left(\overline{\mathcal{P}_{1}}-\overline{\mathcal{P}_{2}}\right)}, n \neq N,
\end{array}, b_{n}=\left\{\begin{array}{l}
\frac{\overline{\mathcal{P}_{1}} \mathcal{P}_{3}+\overline{\mathcal{P}_{2}} \overline{\mathcal{P}_{3}}}{\left(\overline{\mathcal{P}_{1}}-\overline{\mathcal{P}_{2}}\right) \overline{\mathcal{P}_{3}}}, n=0, \\
\frac{\overline{\mathcal{P}_{1}} \mathcal{P}_{3}}{\left(\overline{\mathcal{P}_{1}}-\overline{\mathcal{P}_{2}}\right) \overline{\mathcal{P}_{3}}}, \quad n \neq 0 .
\end{array}\right.\right.
$$

From the recurrence relation in (20) and its initial value in (21), we can get $\beta_{n} \Psi_{n}$ in terms of $\left\{\Psi_{k}\right\}_{k=1}^{n}$ and $\beta_{0}$ only as

$$
\begin{aligned}
\beta_{n} \Psi_{n}= & a_{n} \Psi_{n}+\sum_{k=1}^{n-1}(-1)^{n-k}\left(b_{k}+a_{k}\right) \Psi_{k} \\
& +(-1)^{n}\left(\beta_{0}+b_{0}\right) .
\end{aligned}
$$

It can be shown that $\sum_{n=1}^{N-1} \beta_{n} \Psi_{n}$ is given as in (24) at the top of the next page. When the BSI is available at the transmitters, the source always accesses the channel when the relay's buffer is empty, i.e., $\beta_{0}=1$. Hence, we can stack our remaining $N$ optimization variables, i.e., $\left\{\Psi_{n}\right\}_{n=1}^{N}$, in a vector form as $\boldsymbol{\Psi}=\left(\Psi_{N}, \Psi_{N-1}, \cdots, \Psi_{1}\right)^{T}$, where $(\cdot)^{T}$ denotes vector transposition. From (24), we can get $\phi$ on the following desired form of a linear-fractional function:

$$
\phi=\frac{\boldsymbol{c}^{T} \boldsymbol{\Psi}+d}{\mathbf{1}^{T} \boldsymbol{\Psi}+1},
$$

where

$$
\begin{aligned}
& \boldsymbol{c}= \begin{cases}(q, a,-b, a,-b, \cdots, a)^{T}, & \text { if } N \text { is even, } \\
(q, a,-b, a,-b, \cdots,-b)^{T}, & \text { if } N \text { is odd, }\end{cases} \\
& q=-\left(\overline{\mathcal{P}_{1}} \mathcal{P}_{3}+\overline{\mathcal{P}_{2}} \overline{\mathcal{P}_{3}}\right), \quad a=\mathcal{P}_{2} \overline{\mathcal{P}_{3}}, \quad b=\overline{\mathcal{P}_{1}} \mathcal{P}_{3}, \\
& d= \begin{cases}\left(\overline{\mathcal{P}_{1}}-\overline{\mathcal{P}_{2}}\right) \overline{\mathcal{P}_{3}}-\overline{\mathcal{P}_{1}}, & \text { if } N \text { is even } \\
\left(\overline{\mathcal{P}_{1}}-\overline{\mathcal{P}_{2}}\right) \overline{\mathcal{P}_{3}}, & \text { if } N \text { is odd, }\end{cases}
\end{aligned}
$$

while 1 is an $N \times 1$ vector whose elements are all equal to unity. Also, the constraints are linear-fractional functions of $\left\{\Psi_{k}\right\}_{k=1}^{n}$. Specifically, from (23) and given $\beta_{0}=1$ we get

$$
\begin{aligned}
\beta_{n} & =\frac{a_{n} \Psi_{n}+\sum_{k=1}^{n-1}(-1)^{n-k}\left(b_{k}+a_{k}\right) \Psi_{k}+(-1)^{n}\left(1+b_{0}\right)}{\Psi_{n}} \\
& =\frac{\boldsymbol{c}_{n}{ }^{T} \boldsymbol{\Psi}+d_{n}}{\mathbf{1}_{\boldsymbol{n}}{ }^{T} \boldsymbol{\Psi}}, \quad \text { for } n=1,2, \cdots, N,
\end{aligned}
$$

where 


$$
\sum_{n=1}^{N-1} \beta_{n} \Psi_{n}= \begin{cases}a_{N-1} \Psi_{N-1}-b_{N-2} \Psi_{N-2}+a_{N-3} \Psi_{N-3}-\cdots+a_{1} \Psi_{1}-\beta_{0}-b_{0}, & \text { if } N \text { is even } \\ a_{N-1} \Psi_{N-1}-b_{N-2} \Psi_{N-2}+a_{N-3} \Psi_{N-3}-\cdots-b_{1} \Psi_{1}, & \text { if } N \text { is odd }\end{cases}
$$

$$
\begin{gathered}
\boldsymbol{c}_{\boldsymbol{n}}=(\underbrace{0, \cdots, 0}_{N-n \text { zeros }}, a_{n},-\left(a_{n-1}+b_{n-1}\right), \cdots,(-1)^{k}\left(a_{n-k}+b_{n-k}\right), \cdots)^{T}, \\
d_{n}=(-1)^{n}\left(1+b_{0}\right), \mathbf{1}_{\boldsymbol{n}}=(\underbrace{0, \cdots, 0}_{N-n \text { zeros }}, 1, \underbrace{0, \cdots, 0}_{n \text { zeros }}) .
\end{gathered}
$$

Note that $\boldsymbol{c}, \boldsymbol{c}_{\boldsymbol{n}}, \mathbf{1}$ and $\mathbf{1}_{\boldsymbol{n}}$ are all $N$-dimensional column vectors. Now, since the objective and constraint functions are linear-fractional functions in $\boldsymbol{\Psi}$, the optimization problem in (14) is now on the form of a linear-fractional program. Linearfractional programs are easily converted to linear programs, and hence, can be efficiently solved using standard numerical linear/convex programming tools. For instance, we used CVX, a MATLAB package for solving convex programs [11]. After obtaining the optimal $\Psi,\left\{\beta_{n}\right\}_{n=1}^{N-1}$ can be directly obtained from (30), while the throughput can be readily computed from (16) and (25).

\section{B. Variant II}

In this variant, the state decrement rate of the birth-death Markov chain as well as the throughput expressions are slightly different. Hence, we follow the same steps of variant I with the following slight modifications, where $\delta_{n, N}$ is substituted by zero for all $n$, while the coefficients of $\Psi_{N}$ are altered. Thus,

$$
q=-\overline{\mathcal{P}_{1}} \mathcal{P}_{3}, \text { and } a_{n}=\frac{\mathcal{P}_{2}}{\overline{\mathcal{P}_{1}}-\overline{\mathcal{P}_{2}}}, \forall n .
$$

Thus, we exactly use the same optimization problem of variant I, with only substituting the values of $q$ and $a_{n}$ from (33) into $c$ and $c_{n}$, respectively.

\section{CSI Only at the Transmitter Side}

For performance comparison purposes, we will briefly discuss the system where the transmitters are only provided with outage state information without BSI. In this case, the source becomes oblivious of the relay's queue state, and hence it cannot adapt its access probabilities accordingly. Specifically, in the event (ii)-(b), since the source cannot distinguish between the different states, $\beta_{n}$ can be only set to a fixed value, $0 \leq \beta \leq 1$, for all $n \in\{0,1,2, \cdots, N\}$. In all events, the unawareness of the source about the queue state will only cause packet transmission and dropping at queue state $N$ instead of remaining silent, which is equivalently accounted for in the given throughput expressions. Hence, the throughput expressions for variant I and II are given, respectively, by

$$
\mathcal{T}_{\mathrm{I}}=\left(\left(\overline{\mathcal{P}_{1}}-\overline{\mathcal{P}_{2}}\right) \overline{\mathcal{P}_{3}} \beta+\overline{\mathcal{P}_{1}} \mathcal{P}_{3}+\overline{\mathcal{P}_{2}} \overline{\mathcal{P}_{3}}\right)\left(1-\epsilon_{N}\right),
$$

and

$$
\mathcal{T}_{\text {II }}=\left(\left(\overline{\mathcal{P}_{1}}-\overline{\mathcal{P}_{2}}\right) \overline{\mathcal{P}_{3}} \beta+\overline{\mathcal{P}_{1}} \mathcal{P}_{3}\right)\left(1-\epsilon_{N}\right)+\overline{\mathcal{P}_{2}} \overline{\mathcal{P}_{3}},
$$

where now the state transition probabilities and the state probabilities are calculated for $\beta_{n}=\beta, \forall n \in\{0,1, \cdots, N\}$. The source can still optimize the value of $\beta$ prior to communication in order to maximize the throughput.

\section{NUMERICAL RESUlTS}

In this section, we numerically evaluate the theoretical results of the proposed scheme, and compare them to those obtained via event-based simulations. In the simulations part, we calculate the end-to-end throughput at the destination after the transmission of $10^{5}$ packets. Since block fading channels are assumed, each packet experiences a different channel gain. For numerical evaluation purposes, we assume herein that the channels are Rayleigh fading. Hence, the channel gain of the $i-j$ link, i.e., $g_{i j}$, is exponentially distributed with mean $\pi_{i j}$. Without loss of generality, we absorb the transmit powers into the channel coefficients, while noise variances are set to unity. The channel gains thus denote the received signal-tonoise ratio over each link. The channel outage probabilities, $\mathcal{P}_{i}=\mathbb{P}\left\{\gamma_{i}<2^{\mathcal{R}}-1\right\}$, can be calculated as

$$
\mathcal{P}_{1}=1-\exp \left(-\frac{2^{\mathcal{R}}-1}{\pi_{s r}}\right), \mathcal{P}_{3}=1-\exp \left(-\frac{2^{\mathcal{R}}-1}{\pi_{r d}}\right)
$$

while $\mathcal{P}_{2}$ can be obtained as in [12] by

$$
\mathcal{P}_{2}=1-\frac{\pi_{s r} \exp \left(-\frac{2^{\mathcal{R}}-1}{\pi_{s r}}\right)}{\pi_{s r}+\left(2^{\mathcal{R}}-1\right) \pi_{r r}} .
$$

We compare the performance of the proposed buffered hybrid HDR/FDR scheme with BSI/CSI with the following schemes: 1) buffered hybrid HDR/FDR with CSI only, 2) bufferless FDR, and 3) bufferless HDR. Both variants of the BSI/CSI hybrid scheme were found to have comparable throughput performance. Hence, we only plot herein the results for variant I for clarity of presentation. The throughput of the conventional bufferless schemes can be calculated as the probability of successful packet transmission (the complement of the outage probability) multiplied by the attempted source rate. The throughput of the bufferless HDR and FDR are hence given, respectively, by

$$
\begin{aligned}
& \mathcal{T}_{\mathrm{HDR}}=2 \mathcal{R} \times \frac{1}{2} \times \overline{\mathcal{P}_{1}^{\mathrm{HDR}}} \overline{\mathcal{P}_{3}^{\mathrm{HDR}}}, \\
& \mathcal{T}_{\mathrm{FDR}}=\mathcal{R} \times \overline{\mathcal{P}_{2}} \overline{\mathcal{P}_{3}}
\end{aligned}
$$

where $\mathcal{P}_{1}^{\mathrm{HDR}}$ and $\mathcal{P}_{3}^{\mathrm{HDR}}$ are calculated from (36) and (37) but with $\mathcal{R}$ replaced by $2 \mathcal{R}$ due to the rate penalty encountered by HDR. Note that providing transmit CSI to bufferless systems does not offer any performance gains when fixed rate transmission is adopted. This is due to the causality of packet forwarding at the bufferless relay, where it is forced to wait for the event when the two hops are simultaneously outage-free to attain successful delivery. We summarize the used simulation parameters in the caption of each figure. Also, for all figures, we use unfilled plot marks with connecting lines to represent the theoretical results, while unconnected filled marks of the same shape are used for the simulations results. 


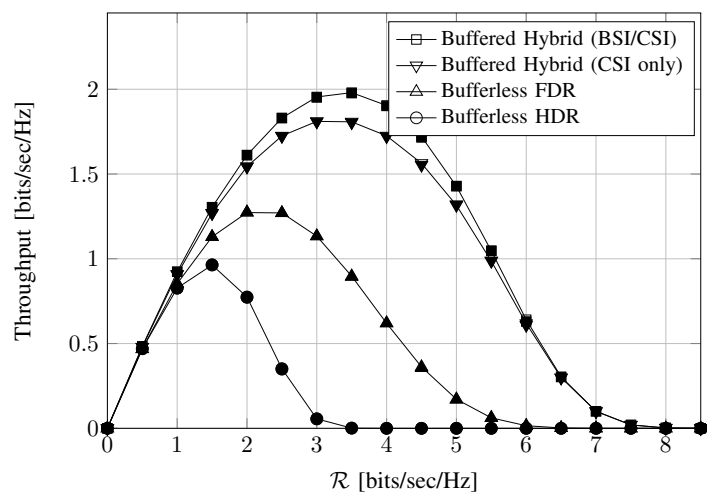

Fig. 3. Throughput vs. source rate, for $\pi_{s r}=\pi_{r d}=15 \mathrm{~dB}, \pi_{r r}=5 \mathrm{~dB}$ and $N=4$ packets.

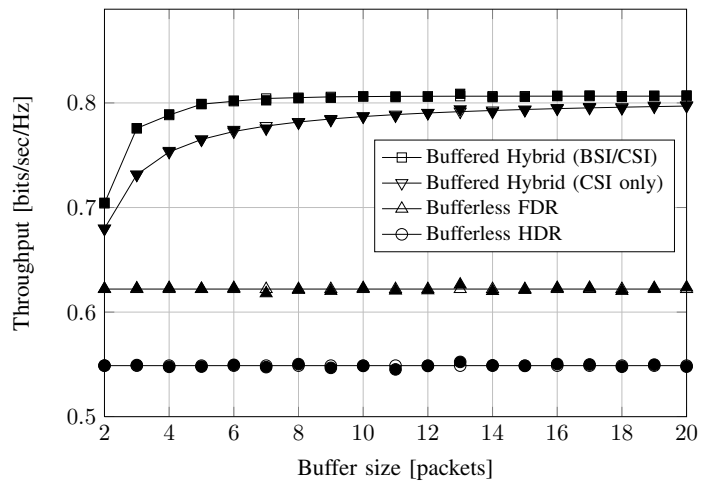

Fig. 4. Throughput vs. buffer size, for $\pi_{s r}=\pi_{r d}=10 \mathrm{~dB}, \pi_{r r}=5 \mathrm{~dB}$ and $R=1 \mathrm{bits} / \mathrm{sec} / \mathrm{Hz}$.

Hence, connected curves with properly filled marks reflect how well-matching the theoretical and simulation results are.

In Fig. 3, we plot the end-to-end throughput versus the source attempted rate $\mathcal{R}$. As shown, the proposed buffered hybrid HDR/FDR scheme when it is only provided with transmit CSI outperforms both bufferless HDR and FDR. This is due to the ability of the scheme to jointly preserve three desirable aspects, namely, 1) the acceptance of additional packets and their storage at the relay's queue when the $r-d$ link experiences outage for possible future forwarding which increases the occupancy of the second hop and hence the throughput, 2) the ability of the full-duplex relay to simultaneously listen and forward which avoids the known rate penalty in HDR, and 3) the agility of the relay to resort to halfduplex operation whenever the self-interference link prevents simultaneous transmission. Further, it can be noticed that providing the source with the BSI can offer further throughput gains. These gains arise from the additionally offered flexibility in link selection, where the source can now avoid transmission whenever the relay's queue is full, and give the opportunity for the relay to drain and recover a room in the queue to accept more packets.

We plot the throughput versus the size of the relay's buffer in Fig. 4. As shown, the throughput of the proposed hybrid scheme with either CSI only or BSI/CSI clearly outperforms that of the bufferless FDR and HDR schemes. Moreover, the throughput gains increase with increasing the buffer size. We can notice that the throughput of the CSI-only scheme approaches that of BSI/CSI as the buffer size increases. This behavior takes place due to the fact that the probability of a full buffer, $\epsilon_{N}$, decreases with buffer size, and the need for the knowledge of BSI at the source diminishes.

\section{CONCLUSION}

In this work, we have proposed a hybrid half-/full-duplex buffer-aided relaying scheme that leverages the available outage and buffer state information to maximize the end-to-end throughput. The formulated optimization problem is put in a linear-fractional program form, which is in turn converted to a linear program that is efficiently solvable using standard numerical linear programming tools. Performance gains are shown to exist when the source is further provided with the relay's buffer state information, compared to systems with only outage state information at the transmitters, especially at low buffer sizes. The exact theoretical paper findings are validated via event-based simulations, which are shown to be in excellent agreement with theory.

\section{REFERENCES}

[1] N. Bhushan, J. Li, D. Malladi, R. Gilmore, D. Brenner, A. Damnjanovic, R. Sukhavasi, C. Patel, and S. Geirhofer, "Network densification: the dominant theme for wireless evolution into 5G," IEEE Commun. Mag., vol. 52, no. 2, pp. 82-89, Feb. 2014.

[2] S. Hong, J. Brand, J. Choi, M. Jain, J. Mehlman, S. Katti, and P. Levis, "Applications of self-interference cancellation in 5G and beyond," IEEE Commun. Mag., vol. 52, no. 2, pp. 114-121, Feb. 2014.

[3] B. Xia, Y. Fan, J. Thompson, and H. V. Poor, "Buffering in a threenode relay network," IEEE Trans. Wireless Commun., vol. 7, no. 11, pp. 4492-4496, Nov. 2008.

[4] N. Zlatanov, R. Schober, and P. Popovski, "Buffer-aided relaying with adaptive link selection,” IEEE J. Sel. Areas Commun., vol. 31, no. 8, pp. 1530-1542, Aug. 2013.

[5] M. Duarte and A. Sabharwal, "Full-duplex wireless communications using off-the-shelf radios: Feasibility and first results," in Proc. ASILOMAR'10, Pacific Grove, CA, Nov. 2010.

[6] J. I. Choi, M. Jain, K. Srinivasan, P. Levis, and S. Katti, "Achieving single channel, full duplex wireless communication," in Proc. ACM MobiCom'10, Chicago, IL, Sept. 2010.

[7] T. Riihonen, S. Werner, and R. Wichman, "Hybrid full-duplex/halfduplex relaying with transmit power adaptation," IEEE Trans. Wireless Commun., vol. 10, no. 9, pp. 3074-3085, Sept. 2011.

[8] M. Khafagy, A Ismail, M.-S. Alouini, and S. Aïssa, "On the outage performance of full-duplex selective decode-and-forward relaying," IEEE Commun. Lett., vol. 17, no. 6, pp. 1180-1183, June 2013.

[9] I. Krikidis, T. Charalambous, and J. S. Thompson, "Buffer-aided relay selection for cooperative diversity systems without delay constraints," IEEE Trans. Wireless Commun., vol. 11, no. 5, pp. 1957-1967, May 2012.

[10] N. Shende, O. Gurbuz, and E. Erkip, "Half-duplex or full-duplex relaying: A capacity analysis under self-interference," in Proc. CISS'13, Baltimore, MD, USA, Mar. 2013.

[11] M. Grant and S. Boyd, "CVX: Matlab software for disciplined convex programming, version 2.1," http://cvxr.com/cvx, Mar. 2014.

[12] T. Kwon, S. Lim, S. Choi, and D. Hong, "Optimal duplex mode for DF relay in terms of the outage probability," IEEE Trans. Veh. Technol., vol. 59, no. 7, pp. $3628-3634$, Sept. 2010. 